\title{
IDENTIFICATION OF THE VALUE PROPOSITION OF AN EVOLVABLE PRODUCTION SYSTEM
}

\author{
Antonio Maffei, Pedro Neves, and Mauro Onori \\ Production Engineering, KTH The Royal Institute of Technology, Stockholm, Sweden
}

\begin{abstract}
Current production paradigms and related biases concerning automation are an obstacle for the technological development and subsequent application of intelligent assembly solutions such as the automation based on the evolvable paradigm. A deeper understanding of the potential behind such technology is a fundamental step towards a proficient industrial embodiment. The concept of Value Proposition can be used as a holistic analytical tool able to support a full characterization of the appeal that such technology has on the assembly automation market. The two dimensional bottom-up approach proposed in this work allows the identification and description of six potential value offerings connected with an Evolvable Assembly system, which in turn pave the way to more efficient business models.
\end{abstract}

\section{INTRODUCTION}

Production engineering research has for decades proposed a wide variety of technological solutions for industry, ranging from the early Flexible Automatic Assembly (FAA) systems [1] to Reconfigurable Manufacturing (RMS) [2, 3], and then from Bionic [4] to Evolvable Production Systems (EPS) [5, 6]. As technology advanced, leading to new materials, pervasive computing and now even cloud technology, it has left industry rather unperturbed: the industrial reality is that if we do not guarantee a financial improvement of some sort, the technology will not be applied.

Initially the promise of flexibility led industry to invest a fair amount of capital in such solutions, with Sony launching its SMART-cell system and the Finnish industry working on its modular assembly concepts (see Flexlink, PMJ Automation, etc.). The results, however, did not meet the promise and since the late 1990's Europe has seen a decline in investment in automation and an ever increasing outsourcing trend.

The inevitable question as to why technology has not succeeded in rooting itself within industry is common yet not very well tackled: as stated earlier, a company must have a firm grasp of what value is generated by a given investment. Up to date, the FAA, RMS or EPS systems have not focused on providing industry with an adequate business model for the corresponding technology. That is an economic model that guides the user into knowing what value is generated, at each stage, of a given technological investment.

This paper will present the basic features of the Value Proposition behind an EPS Business Model, which has been developed for industry through a series of European projects. The Business Model represents a shift in how one may invest in production equipment, and details the expected benefits at each phase of the product lifecycle. The Business Model is presented here in summarised version.

\section{BACKGROUND}

A great deal of literature has been written in the past few years about the erosion of manufacturing's share of the western economies, and the emergence of the service sector as the dominant force in the future economy. Analysis of these trends might lead to the mistaken conclusion that manufacturing is a dying enterprise better left to developing nations where unskilled labour is abundant and plants and equipment are unspoiled by years of use. The fault in this argument lies within the fact that manufacturing represents the real wealth-producing activity of a nation that supports a high standard of living, a fact known years ago and supported by most current roadmaps $[7,8]$.

One of the main reasons why companies have practically abandoned the idea of investing in new production technology is bound to the fact that the business models in use have not changed since the mid-1990's.

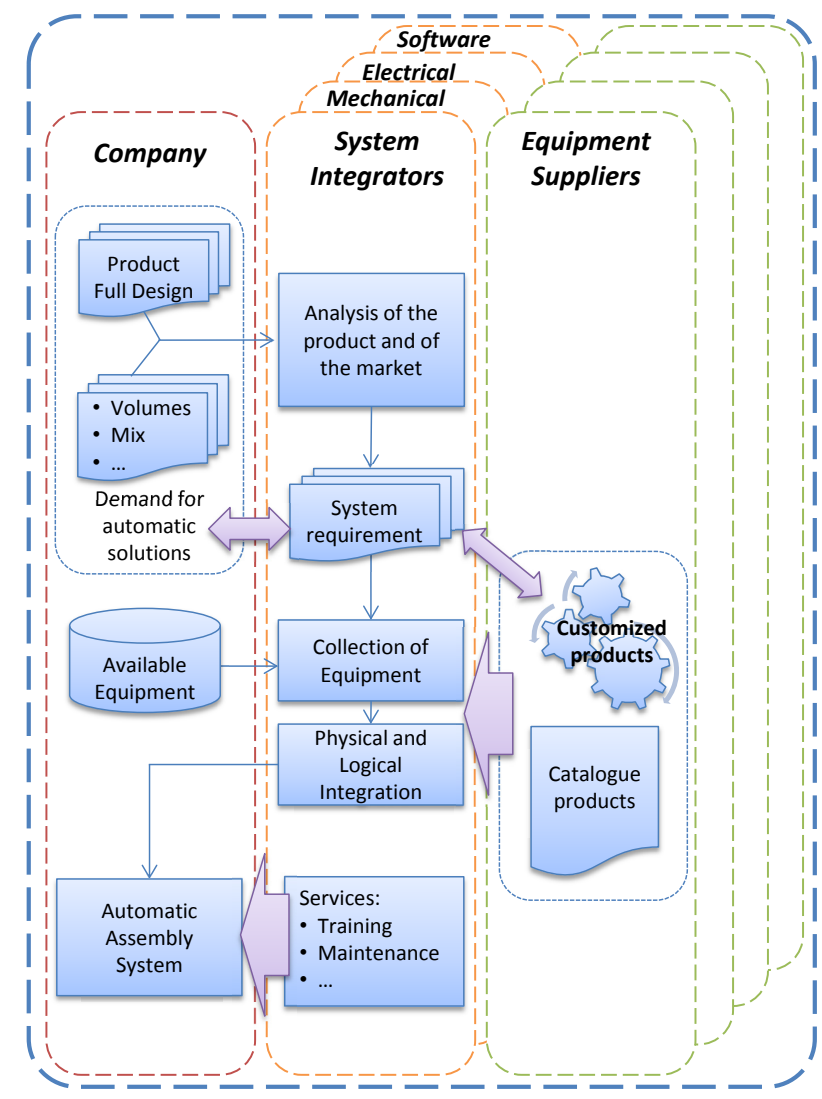

Figure 1. The Engineer-To-Order System Development Approach (Adapted from [9]).

A review of production technology shifts has been detailed by the literature $[10,11]$, illustrating how technology could potentially assist companies in becoming more adaptive, agile or flexible. The issue is that most solutions do not discuss the 
fact that all technology input within a company still follows the Engineer-To-Order principle (ETO). As given in the Figure 1, the existing business model is loosely based on this sequence of transactions. The Engineer to Order business paradigm is embodied through a specific set of stakeholders with welldefined roles in the overall development of the automatic production system.

Noteworthy here is that the categories of stakeholders are well established and contribute to the re-enforcement of the approach: each sector specialises in its role and creates a dependency relation between them. Fundamental however, is the fact that a system is engineered from a given final product design: that is, it is the product details that determine how the assembly system will be and perform [12]. Any future change is included as a potential forecasted characteristic which the system's "flexibility" may cope with. The fact that product details may change even as it is being launched is almost never taken up as a constraining demand, although this is practically standard [9].

The call for more agile and sustainable solutions along with the increasing potential of technology has pushed to question several aspects of this mainstream approach. This scrutiny gave first the impulse to investigate the modularization of the automatic assembly system. Modular Assembly Systems were among the first to recognise this $[13,14]$. As noticed by [15] the modularization of complex products (and therefore of an automatic production system) allows to move from the current Engineer to Order (ETO) to a more efficient Configure to Order (CTO).

In parallel with these developments, scholars and practitioners have studied and developed ways of embedding intelligence into the manufacturing system in order to make the task autonomous, $[16,17]$ thus arriving at the much discussed plug $\&$ produce concept. This new generation of systems is not yet fully developed but the first industrial case studies have been successfully produced [18]. The new paradigm supporting this advancement can be called "Plug to Order". The authors of the PTO approach, with industry as co-developers, soon realised that the underlying business model needed modification as the traditional stakeholder system would be disrupted.

Fundamentally, this new generation of manufacturing technologies carries a highly disruptive potential if compared with the state of the art: in order to be effectively applied it needs, in fact, not only a thorough development of the technical enabling factors, but also a compelling progress of reassessment of all the supporting mechanisms that a company must put in place to fully exploit the innovation and potential added value. As [5] recognized, this significant step forward is comparable to one of the "paradigm shifts" described by [19]. The previous technology (FAA, RMS) supported the traditional paradigm applied in the automation of assembly systems: integral architectures designed around a product or a product family (ETO). By introducing process-oriented modules that are dedicated to a process and may self-configure, be added/removed without programming, etc. introduces new stakeholders, new values, and an entirely novel approach to system design. Evolvable Systems therefore introduce the new business approach of Plug-To-Order (PTO) [20], as seen in Fig.2.

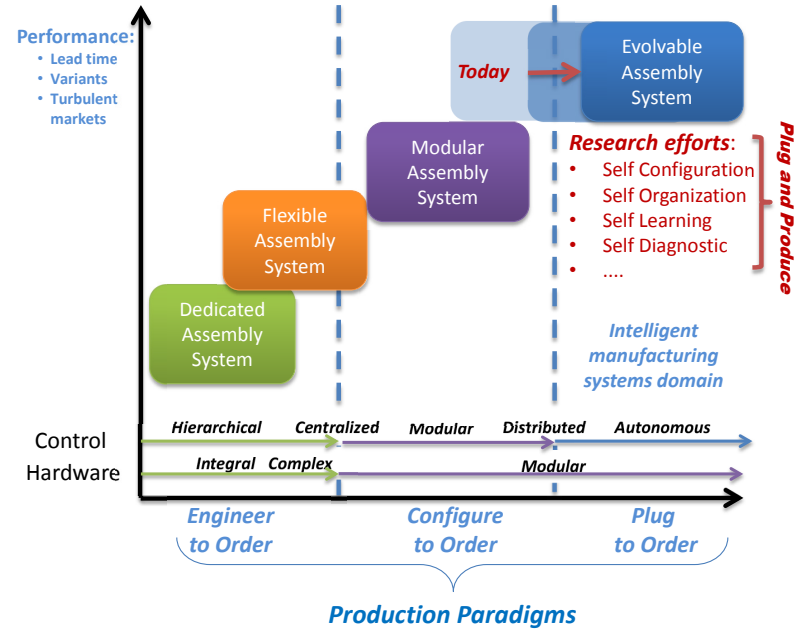

Figure 2. Production Paradigm, From ETO to PTO (adapted from [20])

\section{THE EPS BUSINESS MODEL BASICS}

Companies that adopt automation must really understand the value of it in all its aspects, which entails that if we are to move from ETO to PTO, this must become even more established. In fact the common mistake is to think that automation is a short term investment that can create value only at the moment of using it for a given problem. As some successful companies have shown, however, an automatic plant has the possibility of generating value in all the phases of its life if it is well conceived and designed (see Sony, Toyota, Nokia).

One of the basic steps required is to understand that every step, and each stakeholder, may be a value-generating entity. Any significant technological shift offers interesting possibility to a firm but at the same time it poses serious challenges to its internal organization and relationships with the external environment. The full exploitation of the associated potential can only be achieved if the firm is able to align properly the technological inputs with a coherent business model able to create and capture such value. In addition, the inability of a firm in doing so might even endanger the survival of the company in the first place. An effective business model will detail each value-generating phase and propose a method for implementing it with yield.

Automatic production systems are very complex machine aggregations. Their design, development and use require a very large set of different activities that call for different competences. It is therefore not possible to consider an assembly system as the product of a single entity or activity. The underlying aspect is that if it consists of several entities, as they do in reality, each entity involved must have its profits. This is where the "value proposition" comes into play: defining how each involved entity may gain from this activity. To define this and create the methods by which we generate value-adding methods is, in essence the business model which we need to develop.

As defined in earlier publications [21, 22], EPS are technologically advanced systems: complex in understanding them but, theoretically, easy to run. Therefore, in order to clearly identify all the different value propositions carried by an evolvable assembly system it is therefore necessary to account for all the activities required to design, develop and run them.

In order to encompass all the elementary offerings associated with such installation the scrutiny has been conducted along two fundamental dimensions: 
- Space. Explicit or latent offering must be disclosed for all the tangible and intangible elements that compose an evolvable assembly system. The identification of such elements is presented in the next paragraph. The analysis has been limited to the aspects that characterize an EAS: generic supporting activities or resources have been left out because not relevant for the purpose of this work.

- Time. New value offerings for the same element can emerge in different stages of an EAS's lifecycle. The five phases considered in this enquiry are derived from [23]'s classification of the lifecycle of a value proposition. In detail:

$\circ$ Value Creation. When the element is physically created.

$\circ$ Value Purchase. The process of transferring the element from the supplier (creator) to the user.

- Value Use. Utilization of the element.

$\circ$ Value Renewal. Update of the functionalities of the element.

- Value Transfer. Transfer of the element to another user, or dismissal.

This method has also the advantage of creating a clear classification of the activities that will be the basis of the following Business Model. Figure 3 attempts to briefly summarise the problem: at each stage there are potential value propositions that are yet undefined. This entails that for each stakeholder there will be no supporting business models for decision-making. The first step, therefore, is to clarify which are the elements in an EPS that create differentiated value propositions.

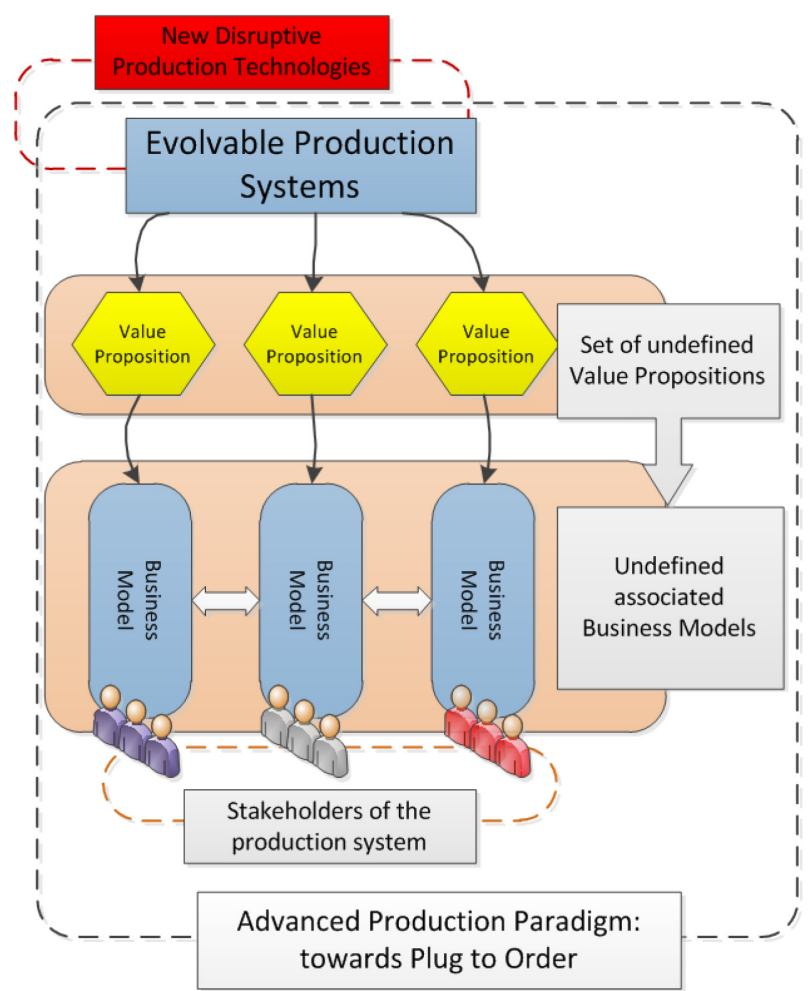

Figure 3. The Basic EPS Business Problem

\section{IDENTIFICATION OF THE CHARACTERIZING ELEMENTS OF AN EPS}

Evolvable Production Systems, as described earlier, are complex systems with modular, embedded equipment. Although, due to their disruptiveness, EPSs have still not found a clear pattern to the market, the current level of development allows identifying the elements that carry independent potential value offerings. Figure 4 provides an overview of such constructs and their physical and logical interconnection.

The main reference for the classification of the elements of an Evolvable Assembly System (EAS, the subset of EPS analysed hereby) is the outcome of the IDEAS project [24]. The EPS paradigm revolves around the concept of Mechatronic Agent, (MA). In general, for the purpose of this work, each MA can be considered as the composition of three elements: an (1) Agent which is a piece of software able to foster a series of elementary behaviors. Those behaviors allow the agent to interact with other agents and exploits some (2) Skills. Skills are conceptual resources strictly related to one or more pieces of (3) Hardware that is the physical representation of the MA. Given the MA nature of hybrid hardware/software entity the controller which allocates the agent and the related skills is intended to be embedded with the rest of the HW.

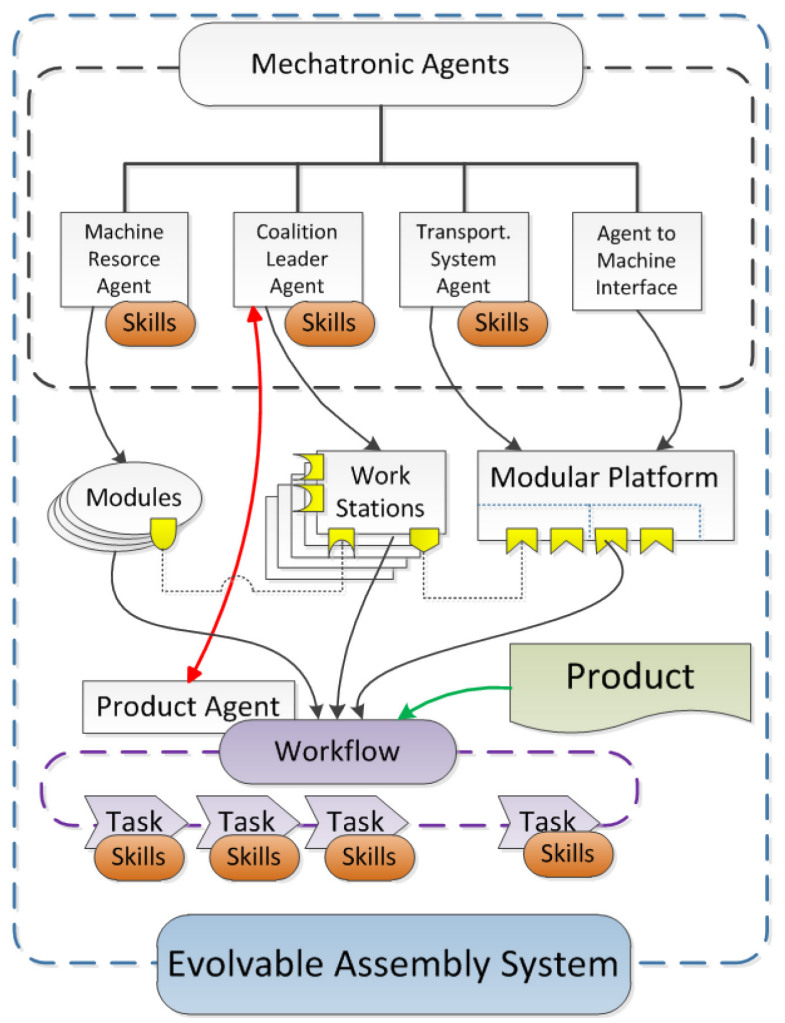

Figure 4. Overview of an EPS: elements and interconnections

Summarizing we can identify the 6 elements that compose an EAS and that therefore will be the basis of the analysis:

1. Multi-Agent System (MAS). It is the "Operative System" of an EPS. It is a modular piece of software that can be physically distributed according to the specific system requirements. It includes all the generic behaviors able to underpin a correct exploitation of the Skills.

2. Skill. Basic construct of the EPS paradigm. Skills are the building blocks of both the EPS process model, identified in the workflow, and of the EPS hardware identified by the platform, the workstation and the modules. Skills are used by the agents and they enclose the necessary information for public interfacing as well as dynamic links with the system's low level libraries (specific of each mechatronic entity).

3. Workflow. Basic construct that represent the whole set of skills and related logical configuration necessary to assemble a product. A workflow is in essence the hierarchically highest composite skill, thus it is governed 
by a Coalition Leader Agent (CLA). The Workflow is designed by the user in function of the process requirement related to the product and the available resources in the system.

4. Modular Platform. Hardware construct governed by the Transport System Agent (TSA). The platform is composed by the repetition of standard modules featuring: (a) interfaces for other platform modules, (b) standard slots for the workstations and (c) logistic between the different slots. If in the system there is requirement for non EPS hardware this needs to be integrated with the platform.

5. Workstation. It is a particular point in the system where one or more tasks are executed. From the hardware point of view it is a collection of one or a more modules. It is governed by a CLA based on processes designed by the user: no further logical integration is required. However, the modules composing a WS need to be physically integrated. A workstation is able to provide the material execution of skills at the intermediates hierarchical level in the related workflow.

6. Module. Construct that embodies the hardware representation of a Machine Resource Agent (MRA). A module is able to provide the material execution of a skill at the lowest hierarchical level in the related workflow.

The elements individuated cover all the relevant activities to be carried on when setting on a new automatic assembly system. It is important to remark that, as for any categorization activity, the final purpose of the devised classification has been an utmost driver in establishing the elements themselves. The aim of this process is to put in evidence all the different components of an EAS that might carry an independent value proposition.

\section{THE EPS VALEU PROPOSITION AS A SET OF OFFERINGS}

The double perspective, spatial and temporal allows at this point plotting the identified value proposition in the following matrix in figure 5 .

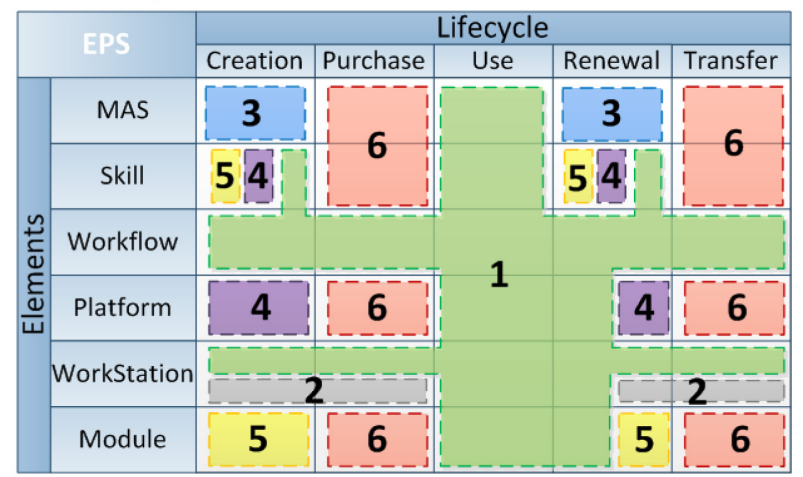

Figure 5. Summary of the identified EPS Value Propositions

With Reference to Figure 5 it is possible to describe 6 general EPS value offerings:

End-User (Green area $\mathbf{n}^{\circ} \mathbf{1}$ ): The value offering that an evolvable production system provides for the user $\left(\mathrm{VO}_{\text {end-user }}\right)$ reaches beyond the simple exploitation of the use phase in the lifecycle of its elements. The entire set of atomic value offerings connected with the workflow has been allocated on $\mathrm{VO}_{\text {end-user }}$. The reason for that lies in the fact that such element is a product-oriented construct which consequently carries value only for that specific production. Besides, once the system is deployed the configuration and reconfiguration of the workflow is, in principle, an easy drag\&drop like task which is manageable also for company with scarce automation expertise. Ultimately the workflow might embed some of the core competencies of the firm which thus has all the strategic interest in keeping it inside. In EPS domain a workflow is conceptually analogous to the highest composition of the skill. This, in turn, means that the end-user plays a role also for the atomic value offerings related to skill. Analogously to the renewal of the workflow value, an EPS end-user is also partially involved in the renewal of the value of modules, platform and workstations.

Workstation Supplier (Grey Area $\mathbf{n}^{\circ}$ 2): Often the end-users which require automatic production systems have no gain in owning all the necessary expertise connected with such installations within their organization charts. Thus such firms must, even in EPS domain, outsource the development of the workstation to specialized business partners.

Multi-Agent System Supplier (Blue Area ${ }^{\circ} 3$ ): In the domain of this dissertation a MAS is a piece of software that provides the basic behaviors for an effective exploitation of the skills as well as the structure for a rational interaction among them. The evolvable paradigm endorses the use of a generic Multi-Agent platform able to cope with any kind of piece of hardware and process through to the related skills. Such premises lead to a very open value offering related to this element. Once the standards for the definition of the skills and the interfacing with the hardware are known the MAS can be created independently.

Platform (Purple area $n^{\circ} 4$ ) and Module (Yellow area $n^{\circ} 5$ ) Suppliers: The evolvable assembly system internal logistic requirements are handled through the aggregation of several kinds of simple logic units which provide the necessary atomic skills connected with the transport domain. The resulting modular platform connects all the workstations currently active in the system through a set of specific composite skills aptly generated as result of the aforementioned aggregation. Given its nature of general purpose element and provided that standards related to the different application are available and implemented the value offered by platform creation can be exploited independently from the temporally subsequent phases in its lifecycle. In the same way platform units are aggregated to create the system that provide the necessary internal logistic to an EPS, the modules are combined into the workstations that seats in the hubs of such network. Consequently the suppliers of the modules can, in principle, exploit a very similar value offering.

Mechatronic Agent Provider (Red area ${ }^{\circ}$ 6): The innovative way of engineering the system introduced by the evolvable paradigm extends the value of the general purpose equipment beyond a single productive cycle. Automatic assembly systems are no longer prototypic installations which the re-engineering costs are, in some extreme cases, higher than the costs of building a new system from scratch: they rather are rapidly deployable and re-deployable sets of standard components (mechatronic agents) that therefore keep a high value throughout different production cycles. The transfer of the value at the end of each use is fundamental in EPS, while in traditional high speed automation was basically identifiable in the cost of dismissing the system and when possible cash from legacy components.

\section{DISCUSSION AND CONCLUSIONS}

The problem of deciding the convenience of an automatic assembly system is not within the scope of this work. A complete manufacturing strategy will, of course, include the possibilities of outsourcing the assembly or using a manual 
assembly system or an hybrid system, but given the already broad target domain the research in this work has been narrowed down to the downstream issues with respect to the decision to employ an AAS. Even if the main object of the analysis is how to bring to market an innovative production system, the authors refrain from dealing with general production innovation in general. The focus herewith remains on "what" a company should do to profit from such a system, rather than "how" to bring to market a product.

As for generalizing the result of this analysis, one can say that this work prove that any major innovation in the production system domain, any advancement that in other words carry a different value proposition with respect to the previous approach, needs an afterthought and consequent re-engineering of the associated business model. Integrated efforts from different firms or clusters of firms will lead to a first-onewinning result in the form of being able to impose their standard.

This work introduces the issue that modular production systems built upon distributed control have a completely different value proposition with respect to the traditional automatic systems based on a rigid integrated architecture and centralized control. This does not mean that they are superior for all the production scenarios and requirement. Fixed automation, as well as Flexible automation still carries an important value proposition that makes them attractive for a very large share of industrial application.

This work also suggests that the EPS paradigm supports the shift from the traditional business model in which the production system is owned by the company that uses it to a more efficient and profitable one that can exploit the financial and operative flexibility of a solution based only on buying the rights for using the system. This improvement is a direct consequence of the two main features of an EPS: modularity and distributed control. Generic and reusable modules that start producing efficiently after simply being plugged into a standard platform foster, in fact, better possibilities to easily transfer their value even across different end users.

Scholars have provided several different hypotheses on how, from a purely qualitative point of view, the disruptive technology can enter in the market. Among them the most interesting are presented by [25] which advocates the use of a new specific market segment that acts as a pivot on the mass of mainstream customer, and [26] that instead sees the innovation first established in the highest end of the existing market and then slowly expand to the mass market. Even though this work has not specifically tested these hypotheses, the study performed on the impact of the EPS paradigm in automatic assembly domain indicates that those scenarios are not incompatible. In detail, the EPS system can serve already existing markets with new unrequired application: this would start a new market indeed, but inside the existing one. It is the case for example of companies that could benefit from agentifing legacy automation equipment.

\section{REFERENCES}

P. L. Nemetz and L. W. Fry, "Flexible manufacturing organizations: implications for strategy formulation and organization design," Academy of Management Review, pp. 627-638, 1988.

[2] H. A. ElMaraghy, "Flexible and reconfigurable manufacturing systems paradigms," International journal of flexible manufacturing systems, vol. 17, pp. 261-276, 2005.

[3] Y. Koren, U. Heisel, F. Jovane, T. Moriwaki, G. Pritschow, G. Ulsoy, and H. Van Brussel, "Reconfigurable manufacturing systems," CIRP
Annals-Manufacturing Technology, vol. 48, pp. 527540, 1999.

[4] K. Ueda, "A concept for bionic manufacturing systems based on DNA-type information," 1992, pp. 853-863.

[5] M. Onori, "Evolvable assembly systems-a new paradigm?," in 33rd International Symposium on Robotics, Stockholm, Sweden, 2002, pp. 617-621.

[6] A. Maffei, K. Dencker, M. Bjelkemyr, and M. Onori, "From Flexibility to Evolvability: ways to achieve self-reconfigurability and full-autonomy," 2009, pp. 74-79.

[7] F. Jovane, E. Westkämper, and D. Williams, The ManuFuture Road: Towards competitive and sustainable high-adding-value manufacturing vol. 10: Springer, 2008.

[8] EUPASS-ROADMAP, "EUPASS Adaptive Assembly Roadmap 2015 deliverable 1.5 f, Project Report-Public Document 1.5 f," NMP-2-CT-20045079782008.

[9] A. Maffei, "Evolvable production systems: foundations for new business models," Lic. Eng., Production Engineering, KTH ITM, Stockholm, 2010.

[10] P. Ferreira, N. Lohse, and S. Ratchev, "Multi-agent architecture for reconfiguration of precision modular assembly systems," Precision Assembly Technologies and Systems, pp. 247-254, 2010.

[11] Z. Bi, S. Lang, W. Shen, and L. Wang, "Reconfigurable manufacturing systems: the state of the art," International Journal of Production Research, vol. 46, pp. 967-992, 2008.

[12] D. Semere, M. Onori, A. Maffei, and R. Adamietz, "Evolvable assembly systems: coping with variations through evolution," Assembly Automation, vol. 28, pp. 126-133, 2008.

[13] G. Rogers and L. Bottaci, "Modular production systems: a new manufacturing paradigm," Journal of Intelligent Manufacturing, vol. 8, pp. 147-156, 1997.

[14] G. Erixon, "Modular function deployment," KTH, 1998.

[15] M. Kratochvil and C. Carson, Growing modular: mass customization of complex products, services and software: Springer Verlag, 2005.

[16] A. Maffei, M. Onori, P. Neves, and J. Barata, "Evolvable Production Systems: Mechatronic Production Equipment with Evolutionary Control," Emerging Trends in Technological Innovation, pp. 133-142, 2010.

[17] P. Neves, "System Evaluation and Learning in Evolvable Production Systems," Licentiate, Production Engineering, The Royal Institute of Technology, Stockholm, 2012.

[18] M. Onori, N. Lohse, J. Barata, and C. Hanisch, "The IDEAS project: plug \& produce at shop-floor level," Assembly Automation, vol. 32, pp. 124-134, 2012.

[19] T. S. Kuhn, "The Structure of Scientific Revolutions," 1962.

[20] A. Maffei, "Characterisation of the Business Models for Innovative, Non-Mature Production Automation Technology," PhD, Production Engineering, Royal Institute of Technology, Stockholm, 2012.

[21] P. Ferreira, "An agent-based self-configuration methodology for modular assembly systems," University of Nottingham, 2011.

[22] A. Maffei and A. Hofmann, "From flexibility to true Evolvability: An introduction to the basic requirements," 2010, pp. 2658-2663. 
[23] A. Osterwalder, "The business model ontology: A proposition in a design science approach," Academic Dissertation, Universite de Lausanne, Ecole des Hautes Etudes Commerciales, vol. 2, 2004.

[24] IDEAS, "Instantly Deployable Evolvable Assembly Systems," 2010-2013.

[25] C. M. Christensen, The innovator's dilemma: when new technologies cause great firms to fail: Harvard Business Press, 1997.

[26] J. M. Utterback and H. J. Acee, "Disruptive technologies: an expanded view," International Journal of Innovation Management, vol. 9, pp. 1-17, 2005. 